\title{
Power Quality Monitoring
}

\author{
Kazem Mazlumi \\ Zanjan University, Zanjan \\ Iran
}

\section{Introduction}

Power quality is the measure, analysis, and improvement of a load bus voltage, to maintain that voltage to be a sinusoid at rated voltage and frequency. The phenomena related to power quality are generally physical stochastic events in the sense that in many cases are appearing and disappearing arbitrarily. Therefore, power quality measure is more than a simple measurement of an electrical parameter; i.e. it is necessary to record them over an enough long time interval. In order to reduce the huge amount of data by recording and analyzing several electrical parameters over a long period of time, some recording limits are set. If these limits are exceeded, the monitoring instruments evaluate the raw data and record only the essential data of the important events.

There are several reasons for monitoring power quality. The most important reason is the economic damage produced by electromagnetic phenomena in critical process loads. Effects on equipment and process operations can include malfunctions, damage, process disruption, and other anomalies.

It is noted that monitoring, alone, is not the solution for power quality problems. In order to solve the power quality problems, some other remedies more than the installation of power quality monitors are needed. In fact, monitoring provides the essential data which are needed for the improvement of power quality. In many projects related to finding a solution for power quality problems, monitoring plays a decisive role, and, therefore, managing monitoring properly helps to minimize the cost of solving problems.

The recorded power quality data depends on the way the instruments record the disturbance levels and how the signals are interpreted. In order to have a correct interpretation of the recorded data, users need to know the specifications of the monitoring instruments such as sampling rate, accuracy, resolution, anti-aliasing filter. The misinterpretation may result in non-existent errors and recording disturbances which in turn may lead to incorrect conclusions and costly decisions.

Effective monitoring programs are important for power reliability assurance for both utilities and customers. It is worth pointing out that most customer power quality problems originate within the customer facility. Monitoring power quality ensures optimal power system performance and effective energy management. Voltage sags, harmonics, interruptions, high-frequency noise, etc., are the most important power quality problems which are seen in industrial and commercial installations. Troubleshooting these problems requires measuring and analyzing power quality and that leads to the importance of monitoring instruments in order to localize the problems and find solutions. Although the 
power quality monitoring relies on measuring various parameters, only voltage sags and locating their origins are studied in this chapter for brevity.

\section{Voltage sag}

Voltage sags are short duration reductions in rms voltage. According to the standards, voltage sag is "a decrease in voltage at the power frequency for durations of half a cycle to 1 minute". The voltage sag is a multi-dimensional disturbance; i.e. the voltage sag is mainly characterized by duration and retained voltage. The duration of a voltage sag is the amount of time during which the voltage magnitude is below the sag threshold. The retained voltage of the voltage sag is the lowest rms voltage in any of the three phases.

Voltage sags are mainly caused by short circuits, starting (or re-accelerating) of large motors, and transformer energizing. It is noted that when an induction motor starts, it can draw very high currents until the rotor comes up to speed. Many disturbances are due to normal operations such as switching loads and capacitors or faults and the opening of circuit breakers to clear faults. Faults are usually caused by natural or accidental events outside the utility's control such as lightning, birds flying close to power lines and getting electrocuted, and trees or equipment contacting power lines.

Several random factors are involved in the analysis of voltage sags. Some of them are listed as follows:

- Fault type: Three-phase faults are more severe than single-phase faults, but the latter happen more frequently.

- Fault location: Faults originated in transmission systems cause sags which can be seen tens of kilometers away.

- $\quad$ Fault impedance: Solid faults cause more severe sags than impedance faults.

- Fault duration: Self-cleared faults cause sags whose duration depends on the fault itself, not on the protection setting.

- Power system modifications: The impedance between the point of observation and the fault point affects the magnitude of the fault caused sag.

As mentioned before, the drop in voltage during a sag may be due to a short circuit being present in the system. The moment the short circuit fault is cleared by the protection system, the voltage starts to return to its original value. Thus, the duration of a sag is determined by the fault clearing time. However, the actual duration of a sag is normally longer than the fault clearing time.

The commonly used definition of sag duration is the number of cycles during which the rms voltage is below a given threshold. This threshold is somewhat different for each monitor but typical values are around $90 \%$ of the nominal voltage. A power quality monitor typically calculates the rms value once every cycle. The main problem is that the so-called post-fault sag affects the sag duration. When the fault is cleared, the voltage does not recover immediately. This is mainly due to the re-energizing and re-acceleration of induction motor load. This postfault sag may last several seconds, much longer than the actual sag. Therefore, the sag duration as defined before, is no longer equal to the fault clearing time. More seriously, different power quality monitors give different values for the sag duration. As the rms voltage recovers slowly, a small difference in threshold setting may already lead to a serious difference in recorded sag duration. Faults in transmission systems are usually cleared faster than faults in distribution systems. In transmission systems, the critical fault clearing time is rather small. 
Thus, fast protection and fast circuit breakers are essential. Also, transmission and subtransmission systems are normally operated as a grid, requiring distance protection or differential protection, both of which allow for fast clearing of the fault. The principal form of protection in distribution systems is over-current protection. This requires a certain amount of time-grading, which increases the fault clearing time. An exception is formed by systems in which current-limiting fuses are used. These have the ability to clear a fault within one halfcycle. In overhead distribution systems, the instantaneous trip of the re-closer leads to a short sag duration, but the clearing of a permanent fault gives a sag with much longer duration.

One of the interests in voltage sags is due to the problems they cause on several types of equipments. Some of the equipments trip when the rms voltage drops below $90 \%$ for longer than one or two cycles. Such the equipments trip tens of times a year. A voltage sag is not as damaging to industry as a interruption, but as there are far more voltage sags than interruptions, the total damage due to sags is still larger. Another important aspect of voltage sags is that they are hard to mitigate. Short and long interruptions can be prevented via simple measures in the local distribution network. Voltage sags at equipment terminals usually is due to short-circuit faults hundreds of kilometers away in the transmission system. It is clear that there is no simple method to prevent the sags. In this chapter we consider all of the sags originate with the faults.

The magnitude of voltage sag is determined from the rms voltage. There are various ways of obtaining the sag magnitude from the rms voltages. Most power quality monitors take the lowest value obtained during the event. As sags normally have a constant rms value during the deep part of the sag, using the lowest value is an acceptable approximation. The sag is characterized through the remaining voltage during the event. This is then given as a percentage of the nominal voltage. Thus, a $90 \%$ sag in a $400 \mathrm{kV}$ system means that the voltage dropped to $360 \mathrm{kV}$.

Fault location also affects the level of the voltage sags on different parts of the network. For example, consider the distribution network shown in Fig. 1, where F1 through F4 indicate fault positions and B1 through B4 indicate the loads. A fault in fault position F1 causes serious sag for the substation bordering the faulted line. This sag is transferred down to all customers fed from the substation A1. As there is normally no generation connected at lower voltage levels, there is nothing to keep up the voltage. The result is that all customers (B2, B3, and B4) experience serious sag. A fault at position F2 does not cause much voltage drop for customer B1. The impedance of the transformers between substation A1 and substation A2 is large enough to considerably limit the voltage drop at high-voltage side of the transformer. The fault at position F2, however, causes a deep sag at substations A3 and A4 and thus for all customers fed from here (B3 and B4). A fault at position F3 causes an interruption for customer B4 when the protection clears the fault. Only customer B3 experiences serious sag whereas customer B1 does not probably sense anything from this fault. To calculate sag magnitude in radial systems, shown in Fig. 2, a method (the voltage divider method) is introduced now. Where $Z_{S}$ is the source impedance at the Point of Common Coupling (PCC), and $Z_{\mathrm{L}}$ is the impedance between the PCC and the fault point. The PCC is the point from which both the fault and the load are fed. In the voltage divider method, the voltage at the PCC is found as follows:

$$
V_{\mathrm{PCC}}=\frac{Z_{\mathrm{L}}}{Z_{\mathrm{S}}+Z_{\mathrm{L}}}
$$




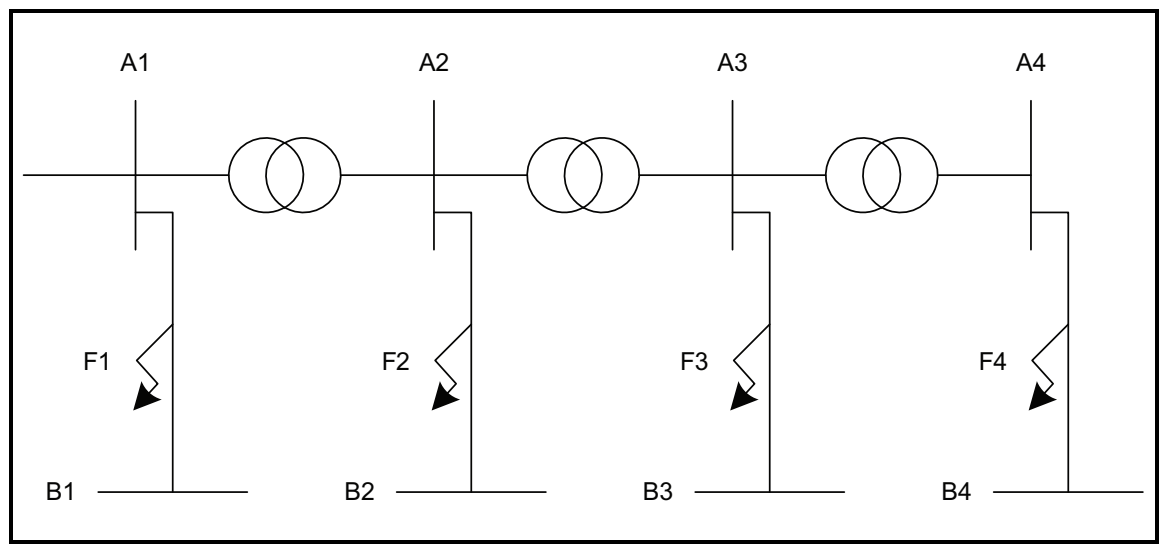

Fig. 1. Sample network for the effects of fault location on voltage sag level

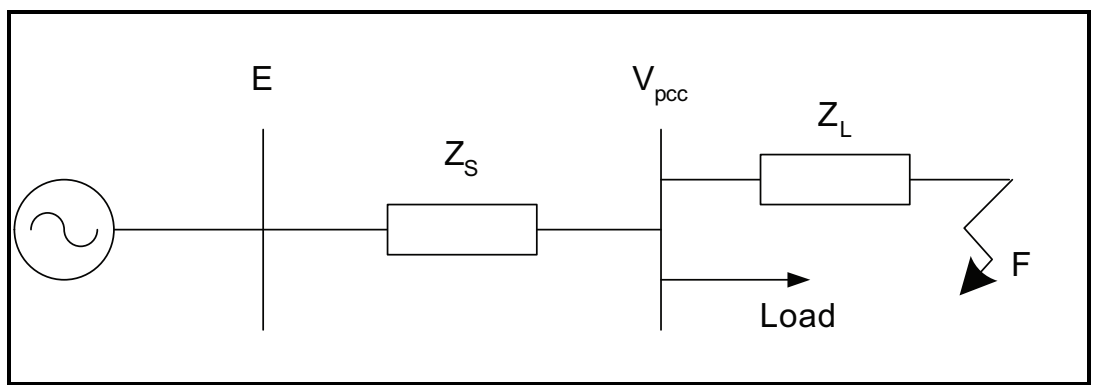

Fig. 2. Voltage sag in a radial network

In Equation (1), the load currents are neglected and it is assumed that the pre-fault voltage is exactly 1 p.u. (e.g., $E=1$ ). Equation (1) shows that the sag becomes more dangerous for faults electrically closer to the customer (i.e., $Z_{\mathrm{L}}$ is smaller), and for weaker systems (i.e., $Z_{\mathrm{S}}$ is larger). It is noted that Equation (1) is suitable for the calculation of the voltage sag in radial networks. For more complicated systems like interconnected transmission systems, matrix calculation is more appropriate. For these cases, the admittance or impedance matrices are used to represent the system.

The voltage sag magnitude is also calculated directly from the fault levels at the PCC and at the fault position. If $S_{\mathrm{FL}}$ and $S_{\mathrm{PCC}}$ are the fault level at the fault position and the PCC, respectively, the sag voltage at the PCC is written as follows:

$$
V_{\mathrm{PCC}}=1-\frac{S_{\mathrm{FL}}}{S_{\mathrm{PCC}}}
$$

The faults in power systems may be symmetrical or unsymmetrical leading to balanced or unbalanced sags, respectively. For the symmetrical faults, only the positive sequence network is required to analyze the during-fault voltage. However the majority of the faults are Single Line to Ground (SLG) faults. The symmetrical components are used to analyze the SLG faults. 


\section{Power quality monitoring}

The process of the power quality analysis consists of four steps, namely, detection, classification, characterization, and location. Power quality monitoring is used to detect the power quality problems. The monitoring solutions depend on the power quality problems being studied. For example, monitoring of the voltage sags caused by remote faults requires a long-time monitoring. To monitor the power quality problems, the instruments such as multi-meters, oscilloscope, disturbance analyzers, flicker meters, and energy monitors may be used. Some other measuring devices are used for monitoring power quality problems. Although the main purpose of these devices is not the monitoring of the power quality problems, they can be used for this reason. Digital Fault Recorders (DFRs), power meters, and etc. are in this category.

DFR is a monitoring device usually used for monitoring the faults related to the power system disturbances. The DFR is used for metering the bus voltages, line currents, and etc. The high sampling rates (between 6 and $10 \mathrm{kHz}$ ) of the DFRs make them possible to record almost all of the events as the transient faults. The DFRs have huge memories for recording and storing the events for a long time.

Monitoring may be used for locating the origins of the power quality disturbances such as voltage sags, harmonics, flickers, and etc. Since the origins of the most voltage sags are the short-circuit faults, the fault locating method and the optimum placement of the monitoring devices are discussed in the rest of this chapter.

\section{Voltage sag measurement}

Once the fault is cleared, the normal service is restored as soon as possible. The permanent short-circuit faults need to repair the causes of the faults. Therefore, finding the fault location of a permanent fault is necessary to remove the fault cause and to re-energize the network. The fault location problem is usually related to the transmission line for which terminal measurements are available. In this case, the objective of the metering is to find the exact fault location.

The retained voltage during the fault gives the magnitude of a voltage sag. Depending on the type of fault that causes the sag, the voltage during the event may be equal or different in the three phases. According to the symmetrical component classification, two types of symmetrical and unsymmetrical voltage sags are recognized.

Three-phase fault causes balanced voltage sag meaning that the phase voltages during the fault are equal in the three phases. For this kind of voltage sags, only one phase voltage is needed to characterize the magnitude and phase angle of the sag. An unsymmetrical fault may cause the sags with the main drop in one phase or two phases. The equations for voltages during the fault are derived for symmetrical and unsymmetrical faults.

\section{Balanced voltage sag}

As mentioned above, a three-phase fault causes balanced voltage sag. Consider a network with $n$ buses and its impedance matrix Z . According to the superposition theorem, the voltage during the fault equals the pre-fault voltage at the bus plus the change in the voltage due to the fault. Therefore, the voltage at bus $k(=1,2, \ldots, n)$ during a three-phase fault at bus $f(=1,2, \ldots, n)$ is given as follows 


$$
v_{k f}=v_{\text {pref }, k}+\Delta v_{k f}
$$

where $v_{\text {pref, } k}$ is the pre-fault voltage at bus $k$ and $\Delta v_{k f}$ is the voltage-change at bus $k$ due to the fault at bus $f$. The $n^{2}$ equations of Equation (3) can be shown in the matrix form as follows

$$
\mathbf{V}_{\mathrm{dfv}}=\mathbf{V}_{\text {pref }}+\Delta \mathbf{V}
$$

The sag matrix $\mathbf{V}_{\mathrm{dfv}}$ contains the during fault voltages $\left(v_{k f}\right)$. For example, the row $k$ of $\boldsymbol{V}_{\mathrm{dfv}}$ contains the retained voltages at bus $k$ when faults occur at buses $1,2, \ldots, f, \ldots, n$. Also, the column $f$ of $\mathbf{V}_{\mathrm{dfv}}$ contains the retained voltage at buses $1,2, \ldots, k, \ldots, n$ for a fault at bus $f$. $\mathbf{V}_{\text {pref }}$ is the pre-fault voltage matrix. Since the pre-fault voltage at bus $k$ is the same for a fault at any bus, the pre-fault voltage matrix is conformed by $n$ equal columns. $\Delta \mathbf{V}$ is a matrix containing the changes in voltage due to faults everywhere. This matrix-based approach is useful for computational implementation of the stochastic assessment.

The voltage changes $\Delta v_{k f}$ are calculated by using the impedance matrix. During a threephase fault at bus $f$, the current injected to the bus $f, i_{f}$, is calculated by

$$
i_{f}=-\frac{v_{\text {pref }, f}}{Z_{f f}}
$$

where $v_{\text {pref, } f}$ is the pre-fault voltage at the faulted bus $f$ and $Z_{f f}$ is the impedance seen looking into the network at the faulted bus $f$. It is noted that only the positive sequence is needed to calculate the fault currents.

By obtaining the injected current due to the fault at bus $f$, the change in voltage at bus $k$ is calculated using the transfer impedance $Z_{k f}$ as follows

$$
\Delta v_{k f}=-Z_{k f} \frac{v_{\text {pref }, f}}{Z_{f f}}
$$

The transfer impedance is the voltage that exists on bus $k$ when bus $f$ is driven by an injection current of unity. Replacing $\Delta v_{k f}$ from Equation (6) in Equation (3) results in

$$
v_{k f}=v_{\text {pref }, k}-Z_{k f} \frac{v_{\text {pref }, f}}{Z_{f f}}
$$

Equation (7) is simplified by neglecting the load, which allows taking the pre-fault voltages equal to $1 \mathrm{p} . \mathrm{u}$. This equation shows that the voltage change at a bus $k$ due to a three-phase fault at bus $f$ is given by the quotient between the transfer impedance and the driven point impedance at the faulted bus. The positive sequence impedance matrix $Z$ is a diagonal dominant full matrix for a connected network. This means that every bus is exposed to sag due to fault everywhere in the network. However, the magnitude of the voltage drop depends on the transfer impedance between the observation bus and the faulted point. Therefore, the load buses are not seriously affected by faults located far away in the system. The voltage change also depends on the driving impedance at the faulted bus. The driving impedance determines the weakness of the bus. 


\section{Unbalanced voltage sag}

Unbalanced voltage sags are caused by unsymmetrical faults. To analyze the unsymmetrical faults the use of symmetrical components is required. Since the sequences in symmetrical systems are independent, Equation (3) can be written for each sequence network and determine the during-fault voltage for each of the sequence components. Before the occurrence of the fault, bus voltages only contain a positive-sequence component, thus prefault voltage matrices of zero and negative sequences are null.

$$
\begin{aligned}
& V_{\mathrm{dfv}}^{0}=0+\Delta V^{0} \\
& V_{\mathrm{dfv}}^{1}=V_{\mathrm{pref}}^{1}+\Delta V^{1} \\
& V_{\mathrm{dfv}}^{2}=0+\Delta V^{2}
\end{aligned}
$$

Once the sequence during-fault voltages are determined the phase voltages are calculated applying the symmetrical components transformation. The superscripts 0,1 , and 2 indicate the zero, positive and negative sequences, respectively. Therefore Equation (9) gives the phase voltages considering phase $\mathrm{A}$ as the symmetrical phase.

$$
\begin{aligned}
& V_{\mathrm{dfv}}^{\mathrm{A}}=V_{\mathrm{dfv}}^{0}+V_{\mathrm{dfv}}^{1}+V_{\mathrm{dfv}}^{2} \\
& V_{\mathrm{dfv}}^{\mathrm{B}}=V_{\mathrm{dfv}}^{0}+a^{2} V_{\mathrm{dfv}}^{1}+a V_{\mathrm{dfv}}^{2} \\
& V_{\mathrm{dfv}}^{\mathrm{C}}=V_{\mathrm{dfv}}^{0}+a V_{\mathrm{dfv}}^{1}+a^{2} V_{\mathrm{dfv}}^{2}
\end{aligned}
$$

Where the superscripts A, B, and C indicate the three phase voltages. By replacing Equation (8) in Equation (9), Equation (10) is written as follows

$$
\begin{aligned}
& V_{\mathrm{dfv}}^{\mathrm{A}}=V_{\mathrm{pref}}^{1}+\Delta V^{0}+\Delta V^{1}+\Delta V^{2} \\
& V_{\mathrm{dfv}}^{\mathrm{B}}=a^{2} V_{\text {pref }}^{1}+\Delta V^{0}+a^{2} \Delta V^{1}+a \Delta V^{2} \\
& V_{\mathrm{dfv}}^{\mathrm{C}}=a V_{\text {pref }}^{1}+\Delta V^{0}+a \Delta V^{1}+a^{2} \Delta V^{2}
\end{aligned}
$$

Equation (10) gives the retained phase voltages during the fault and is independent of the fault type. The only missing part is the change in the voltage during the fault $\Delta V$ for each sequence. The following sub-sections derive these expressions. Equations are derived for a general element $(k, f)$ but matrix expressions are derived from them. All the analysis is for phase $\mathrm{A}$ as the symmetrical phase.

\subsection{Voltage changes in Single Line to Ground (SLG) fault}

SLG faults cause one of the types of the unsymmetrical voltage sags. The short circuit current is found by connecting the sequence networks in series. So the current flowing through the three sequence networks is the same. Thus Equation (11) gives the injected currents in the positive, negative and zero sequence networks.

$$
i_{f}^{0}=i_{f}^{1}=i_{f}^{2}=\frac{-v_{\mathrm{pref}, f}^{\mathrm{A}}}{Z_{f f}^{0}+Z_{f f}^{1}+Z_{f f}^{2}}
$$

The sequence voltage changes at bus $k$ due to the SLG fault at bus $f$ are given by Equation (12). 


$$
\begin{aligned}
& \Delta v_{k f}^{1}=-Z_{k f}^{1} \frac{v_{\mathrm{pref}, f}^{\mathrm{A}}}{Z_{f f}^{0}+Z_{f f}^{1}+Z_{f f}^{2}} \\
& \Delta v_{k f}^{2}=-Z_{k f}^{2} \frac{v_{\mathrm{pref}, f}^{\mathrm{A}}}{Z_{f f}^{0}+Z_{f f}^{1}+Z_{f f}^{2}} \\
& \Delta v_{k f}^{0}=-Z_{k f}^{0} \frac{v_{\mathrm{pref}, f}^{\mathrm{A}}}{Z_{f f}^{0}+Z_{f f}^{1}+Z_{f f}^{2}}
\end{aligned}
$$

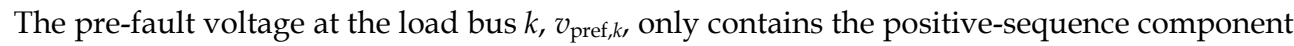
and is equal to the pre-fault voltage in phase A. The during-fault sequence voltages at bus $k$ are given by Equation (13).

$$
\begin{aligned}
& v_{k f}^{1}=v_{\mathrm{pref}, k}^{\mathrm{A}}-Z_{k f}^{1} \frac{v_{\mathrm{pref}, f}^{\mathrm{A}}}{Z_{f f}^{0}+Z_{f f}^{1}+Z_{f f}^{2}} \\
& v_{k f}^{2}=-Z_{k f}^{2} \frac{v_{\mathrm{pref}, f}^{\mathrm{A}}}{Z_{f f}^{0}+Z_{f f}^{1}+Z_{f f}^{2}} \\
& v_{k f}^{0}=-Z_{k f}^{0} \frac{v_{\mathrm{pref}, f}^{\mathrm{A}}}{Z_{f f}^{0}+Z_{f f}^{1}+Z_{f f}^{2}}
\end{aligned}
$$

After transforming the equations into the phase components, Equation (14) is derived.

$$
\begin{aligned}
& v_{k f}^{\mathrm{A}}=v_{\text {pref }, k}^{\mathrm{A}}-\left(Z_{k f}^{1}+Z_{k f}^{2}+Z_{k f}^{0}\right) \frac{v_{\text {pref }, f}^{\mathrm{A}}}{Z_{f f}^{0}+Z_{f f}^{1}+Z_{f f}^{2}} \\
& v_{k f}^{\mathrm{B}}=a^{2} v_{\text {pref }, k}^{\mathrm{A}}-\left(a^{2} Z_{k f}^{1}+a Z_{k f}^{2}+Z_{k f}^{0}\right) \frac{v_{p r e f, f}^{\mathrm{A}}}{Z_{f f}^{0}+Z_{f f}^{1}+Z_{f f}^{2}} \\
& v_{k f}^{\mathrm{C}}=a v_{\text {pref }, k}^{\mathrm{A}}-\left(a Z_{k f}^{1}+a^{2} Z_{k f}^{2}+Z_{k f}^{0}\right) \frac{v_{\text {pref }, f}^{\mathrm{A}}}{Z_{f f}^{0}+Z_{f f}^{1}+Z_{f f}^{2}}
\end{aligned}
$$

From Equation (13), it is clear that the phase voltages at bus $k$ do not contain a zero sequence component if the zero sequence of the transfer impedance $Z_{k f}$ is null. The zero sequence of the transfer impedance is null when the load and the fault buses are at different sides of a transformer with delta winding. Equation (14) gives the retained phase voltage for a general case. The negative sequence impedance matrix is usually equals to the positive sequence impedance matrix. If the zero sequence voltage can be neglected, Equation (14) is simplified as Equation (15).

$$
\begin{aligned}
& v_{k f}^{\mathrm{A}}=v_{\text {pref }, k}^{\mathrm{A}}-\left(Z_{k f}^{1}\right) \frac{v_{\mathrm{pref}, f}^{\mathrm{A}}}{\frac{Z_{f f}^{0}}{2}+Z_{f f}^{1}} \\
& v_{k f}^{\mathrm{B}}=a^{2} v_{\text {pref }, k}^{\mathrm{A}}+\left(\frac{1}{2} Z_{k f}^{1}\right) \frac{v_{\text {pref }, f}^{\mathrm{A}}}{\frac{Z_{f f}^{0}}{2}+Z_{f f}^{1}} \\
& v_{k f}^{\mathrm{C}}=a v_{\text {pref }, k}^{\mathrm{A}}+\left(\frac{1}{2} Z_{k f}^{1}\right) \frac{v_{\text {pref }, f}^{\mathrm{A}}}{\frac{Z_{f f}^{0}}{2}+Z_{f f}^{1}}
\end{aligned}
$$


Equation (15) shows that the retained phase voltages at the observation bus is calculated using a balanced short circuit algorithm by adding a fault impedance equal to half of the value of the zero sequence driving point impedance at the faulted bus. This means that a SLG fault is less severe than a three phase fault in regard to the depression in the voltage seen at the load bus. Due to the zero-sequence impedance, the faulted phase shows a smaller drop compared to a sag originated by a three-phase fault.

\subsection{Voltage changes in Line to Line (LL) fault}

For a LL fault, only the positive and the negative sequence networks are considered to analyze the fault. There is no zero sequence current and voltage. In this type of fault, the positive sequence current at the fault location is equal in magnitude to the negative sequence current, but in opposite direction. Equation (16) gives the injected currents in the sequence networks.

$$
i_{f}^{1}=-\frac{v_{\mathrm{pref}, f}^{\mathrm{A}}}{Z_{f f}^{1}+Z_{f f}^{2}}=-i_{f}^{2}
$$

The change in positive and negative sequence voltages are given by Equation (17). There is no zero sequence voltage change.

$$
\begin{aligned}
& \Delta v_{k f}^{1}=-Z_{k f}^{1} \frac{v_{p r e f, f}^{A}}{Z_{f f}^{1}+Z_{f f}^{2}} \\
& \Delta v_{k f}^{2}=Z_{k f}^{2} \frac{v_{p r e f, f}^{A}}{Z_{f f}^{1}+Z_{f f}^{2}}
\end{aligned}
$$

The retained phase voltages are obtained by adding the pre-fault voltage to Equation (17). Equation (18) shows transforming the sequence components into the phase components.

$$
\begin{aligned}
& v_{k f}^{\mathrm{A}}=v_{\mathrm{pref}, k}^{\mathrm{A}}+\left(Z_{k f}^{2}-Z_{k f}^{1}\right) \frac{v_{\mathrm{pref}, f}^{\mathrm{A}}}{Z_{f f}^{1}+Z_{f f}^{2}} \\
& v_{k f}^{\mathrm{B}}=a^{2} v_{\mathrm{pref}, k}^{\mathrm{A}}+\left(a Z_{k f}^{2}-a^{2} \cdot Z_{k f}^{1}\right) \frac{v_{\mathrm{pref}, f}^{\mathrm{A}}}{Z_{f f}^{1}+Z_{f f}^{2}} \\
& v_{k f}^{\mathrm{C}}=a \cdot v_{p r e f, k}^{A}+\left(a^{2} \cdot Z_{k f}^{2}-a \cdot Z_{k f}^{1}\right) \frac{v_{p r e f, f}^{A}}{Z_{f f}^{1}+Z_{f f}^{2}}
\end{aligned}
$$

If the positive sequence impedance matrix is equal to the negative sequence impedance matrix, Equation (18) is simplified as indicated in Equation (19).

$$
\begin{aligned}
& v_{k f}^{\mathrm{A}}=v_{\text {pref }, k}^{\mathrm{A}} \\
& v_{k f}^{\mathrm{B}}=a^{2} v_{\text {pref }, k}^{\mathrm{A}}+\left(\mathrm{j} \sqrt{3} Z_{k f}^{1}\right) \frac{v_{\text {pref }, f}^{\mathrm{A}}}{2 Z_{f f}^{1}} \\
& v_{k f}^{\mathrm{C}}=a v_{\text {pref }, k}^{\mathrm{A}}-\left(\mathrm{j} \sqrt{3} Z_{k f}^{1}\right) \frac{v_{\text {pref }, f}^{\mathrm{A}}}{2 Z_{f f}^{1}}
\end{aligned}
$$




\subsection{Voltage changes in Double Line to Ground (DLG) fault}

In DLG faults, the sequence networks are connected in parallel to derive the fault current. Equation (20) gives the injected currents in each one of the sequence networks.

$$
\begin{aligned}
& i_{f}^{1}=-\frac{v_{\mathrm{pref}, f}^{\mathrm{A}}}{Z_{f f}^{1}+\frac{Z_{f f}^{0} \cdot Z_{f f}^{2}}{Z_{f f}^{0}+Z_{f f}^{2}}} \\
& i_{f}^{2}=-\frac{Z_{f f}^{0}}{Z_{f f}^{0}+Z_{f f}^{2}} i_{f}^{1} \\
& i_{f}^{0}=-\frac{Z_{f f}^{2}}{Z_{f f}^{0}+Z_{f f}^{2}} i_{f}^{1}
\end{aligned}
$$

Equation (21) gives the retained sequence voltages in a DLG fault.

$$
\begin{aligned}
& v_{k f}^{1}=v_{\text {pref }, k}^{\mathrm{A}}+Z_{k f}^{1} \cdot i_{f}^{1} \\
& v_{k f}^{2}=Z_{k f}^{2}\left(-\frac{Z_{f f}^{0}}{Z_{f f}^{2}+Z_{f f}^{0}} i_{f}^{1}\right) \\
& v_{k f}^{0}=Z_{k f}^{0}\left(-\frac{Z_{f f}^{2}}{Z_{f f}^{2}+Z_{f f}^{0}} i_{f}^{1}\right)
\end{aligned}
$$

Finally, the retained phase voltages are found by applying the symmetrical component transformation. The transforming equations are shown in Equation (22).

$$
\begin{aligned}
& v_{k f}^{A}=v_{p r e f, k}^{A}+\frac{\left(Z_{k f}^{2}-Z_{k f}^{1}\right) Z_{f f}^{0}+\left(Z_{k f}^{0}-Z_{k f}^{1}\right) Z_{f f}^{2}}{Z_{f f}^{1} \cdot Z_{f f}^{0}+Z_{f f}^{2} \cdot Z_{f f}^{1}+Z_{f f}^{2} \cdot Z_{f f}^{0}} v_{p r e f, f}^{A} \\
& v_{k f}^{B}=a^{2} \cdot v_{p r e f, k}^{A}+\frac{\left(a \cdot Z_{k f}^{2}-a^{2} \cdot Z_{k f}^{1}\right) Z_{f f}^{0}+\left(Z_{k f}^{0}-a^{2} \cdot Z_{k f}^{1}\right) Z_{f f}^{2}}{Z_{f f}^{1} \cdot Z_{f f}^{0}+Z_{f f}^{2} \cdot Z_{f f}^{1}+Z_{f f}^{2} \cdot Z_{f f}^{0}} v_{p r e, f}^{A} \\
& v_{k f}^{C}=a \cdot v_{p r e f, k}^{A}+\frac{\left(a^{2} \cdot Z_{k f}^{2}-a \cdot Z_{k f}^{1}\right) Z_{f f}^{0}+\left(Z_{k f}^{0}-a \cdot Z_{k f}^{1}\right) Z_{f f}^{2}}{Z_{f f}^{1} \cdot Z_{f f}^{0}+Z_{f f}^{2} \cdot Z_{f f}^{1}+Z_{f f}^{2} \cdot Z_{f f}^{0}} v_{p r e f, f}^{A}
\end{aligned}
$$

\section{Prediction of voltage sags}

Voltage sags originated by faults have many predictable characteristics. For a three-phase fault the voltage sag matrix is given by

$$
\mathbf{V}_{\mathrm{dfv}}=\mathbf{V}_{\text {pref }}-\mathbf{Z}(\operatorname{diag} \mathbf{Z})^{-1} \mathbf{V}_{\text {pref }}^{\mathrm{T}}
$$

where $\mathbf{Z}$ is the impedance matrix, $\mathbf{V}_{\text {pref }}$ is the pre-fault voltage matrix, and (diagZ) ${ }^{-1}$ is the matrix formed by the inverse of the diagonal elements of the impedance matrix. If the prefault loads are neglected, then voltages before the fault are considered $1 \mathrm{pu}$. Under this assumption, Equation (23) is written as follows 


$$
\mathbf{V}_{d f o}=\mathbf{1}-\mathbf{Z}(\operatorname{diag} \mathbf{Z})^{-1} \mathbf{1}
$$

where $\mathbf{1}$ is a matrix full of ones and such that its dimension is equal to the dimension of $\mathbf{Z}$.

The square $n \times n$ matrix $\mathbf{V}_{\text {dfv }}$ contains the sags at each bus of the network due to faults at each one of the buses. The during fault voltage at a general bus $j$ when a fault occurs at that bus is contained in the diagonal of $\mathbf{V}_{\mathrm{dfv}}$ and is zero for a solid three phase fault. Off-diagonal elements of $\mathbf{V}_{\mathrm{dfv}}$ are the sags at a general bus $k$ due to a fault at a general position $f$. Hence, column $f$ contains the during-fault voltages at buses $1,2, \ldots, f, \ldots, n$ during the fault at bus $f$. This means that the effect, in terms of sags, of a fault at a given bus of the system is contained in columns of the voltage sag matrix. This information is usually presented on the single line diagram of the power system and it is called affected area. A sample $n \times n$ sag matrix is shown as follows

$$
V_{d f o}=\left[\begin{array}{cccc}
0 & 1-\frac{z_{12}}{z_{22}} & \cdots & 1-\frac{z_{1 n}}{z_{n n}} \\
1-\frac{z_{21}}{z_{11}} & 0 & \cdots & 1-\frac{z_{2 n}}{z_{n n}} \\
\vdots & \vdots & \ddots & \vdots \\
1-\frac{z_{n 1}}{z_{11}} & 1-\frac{z_{n 2}}{z_{22}} & \cdots & 0
\end{array}\right]
$$

In this chapter, a real sample network is used for description of the voltage sag matrix. Fig. 3 shows a 41-bus 230-kV of Tehran Regional Electric Company in Iran.

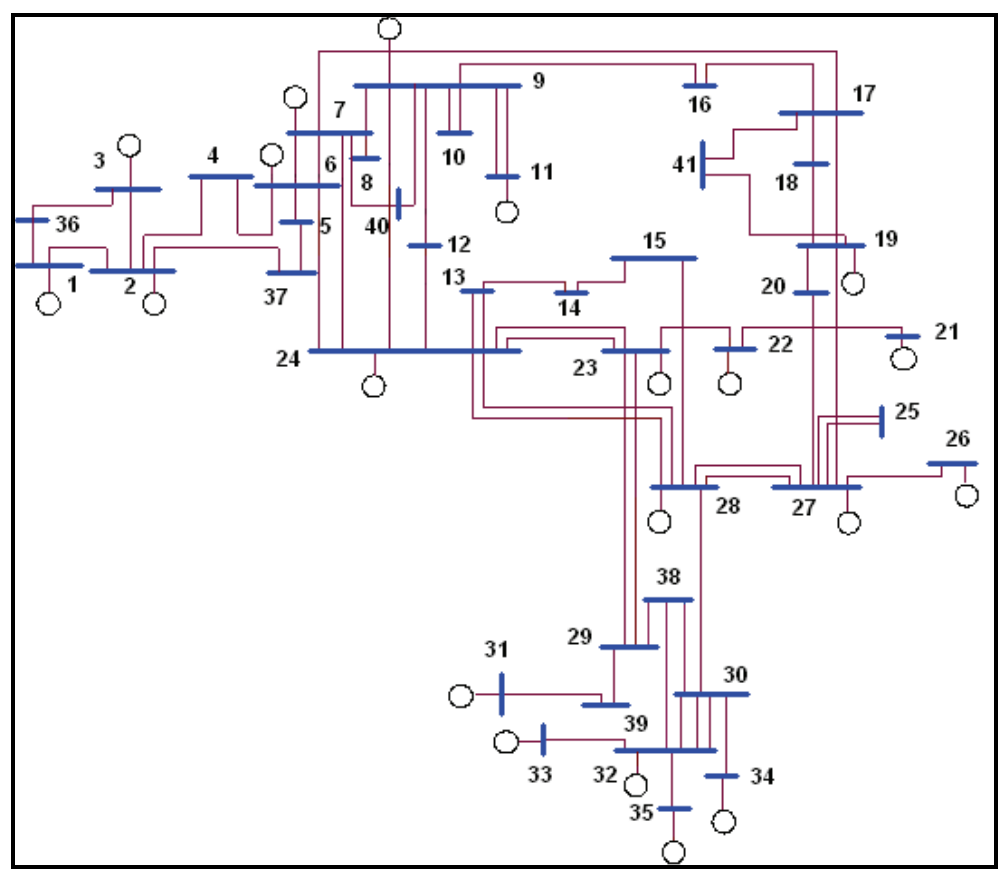

Fig. 3. Single line diagram of a $230 \mathrm{kV}$ real case study 
Applying Equation (23), it is found the during fault voltages for the 41 buses due to the faults on each one of the 41 buses. Equation (26) shows the resulting voltage sag matrix. It is seen that column 34 of the sag matrix contains the during fault voltages at each one of the 41 buses when a three-phase fault occurs at bus 34 . Column 34 of the sag matrix contains the information to draw the affected area of the system due to a fault at bus 34 .

$$
V_{d f v}=\left[\begin{array}{cccccc}
0 & 1-\frac{z_{1,2}}{z_{2,2}} & \cdots & 1-\frac{z_{1,34}}{z_{34,34}} & \cdots & 1-\frac{z_{1,41}}{z_{41,41}} \\
1-\frac{z_{2,1}}{z_{1,1}} & 0 & \cdots & 1-\frac{z_{2,34}}{z_{34,34}} & \cdots & 1-\frac{z_{2,41}}{z_{41,41}} \\
\vdots & \vdots & \vdots & \vdots & \vdots & \vdots \\
1-\frac{z_{34,1}}{z_{1,1}} & 1-\frac{z_{34,2}}{z_{2,2}} & \cdots & 0 & \cdots & 1-\frac{z_{34,41}}{z_{41,41}} \\
\vdots & \vdots & \vdots & \vdots & \vdots & \vdots \\
1-\frac{z_{41,1}}{z_{1,1}} & 1-\frac{z_{41,2}}{z_{2,2}} & \cdots & 1-\frac{z_{41,34}}{z_{34,34}} & \cdots & 0
\end{array}\right]
$$

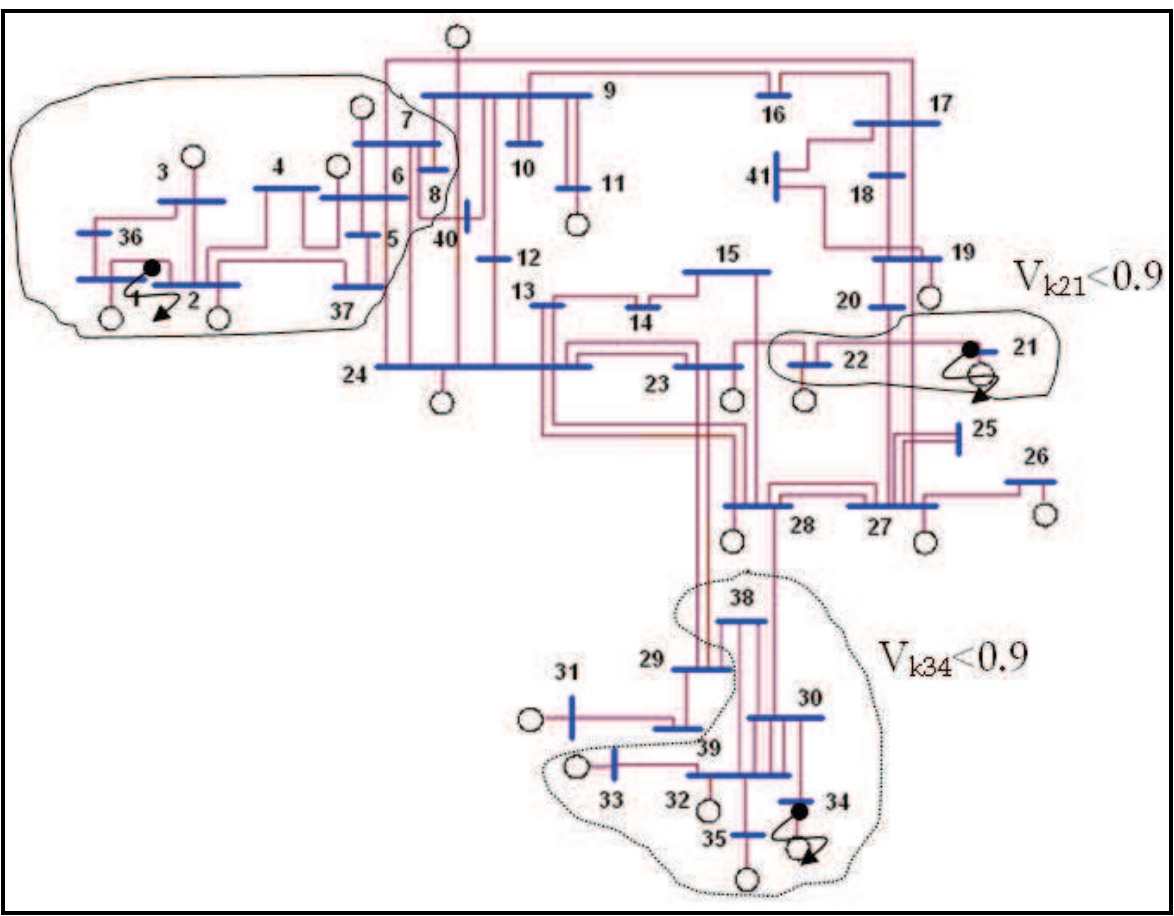

Fig. 4. Affected areas for three-phase fault at buses 34 and 21

The affected area contains the load buses that present a during fault voltage lower than a given value due to a fault at a given bus. In Fig. 4, three affected areas are presented for 
three-phase faults on the middle of the line 1-2 and the buses 21 and 34 . The areas enclose the load buses presenting a sag more severe than a retained voltage of 0.9 p.u. If the threshold is less than 0.9 , the areas are smaller than the areas shown in Fig. 4 . Only the original impedance matrix of positive sequence of the system is used to build the exposed areas. Faults on lines are more frequent than fault on buses of the system, however faults on buses cause more severe sags in terms of magnitude and therefore are considered for building the affected areas. In the rest of this chapter, only faults on the buses are to be considered.

The exposed area (area of vulnerability) is contained in rows of the voltage sag matrix and as in the case of the affected area can be graphically presented on the single line diagram. Fig. 5 presents the exposed area of bus 32. The exposed area encloses the buses and line segments where faults cause a sag more severe than a given value. In Fig. 5, the 0.5 p.u. exposed area of bus 32 contains buses 30 and 32. Similarly, the 0.8 pu exposed area for bus 32 contains all the buses where faults cause a retained voltage lower than 0.8 pu. Fig. 5 suggests that the exposed area is a closed set containing buses.

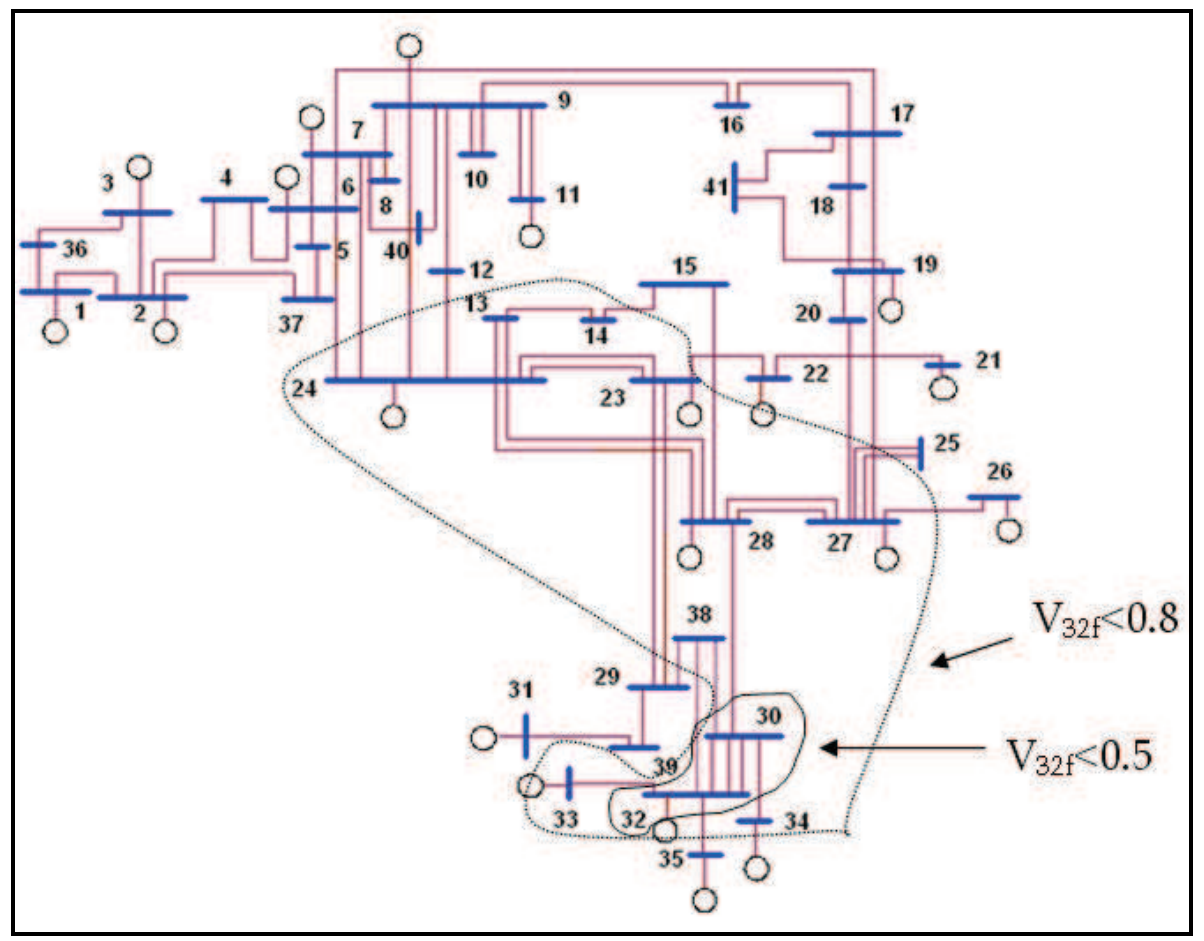

Fig. 5. Exposed area of bus 32 for three-phase faults

Unsymmetrical faults can also be considered to define the exposed area of a sensitive load. Positive, negative, and zero sequence impedance matrices are needed to perform the calculations.

To show the exposed areas for symmetrical and unsymmetrical faults, the exposed areas of a three phase fault and a SLG fault at bus 35 are illustrated in Fig. 6. In this figure, the $0.8 \mathrm{pu}$ 
SLG fault exposed area of bus 35 contains buses 30 and 32. Also, the 0.8 pu three phase fault exposed area contains buses 30,32, and 38. The SLG fault exposed area is almost coincident with the three phase fault exposed area, however, a bit smaller.

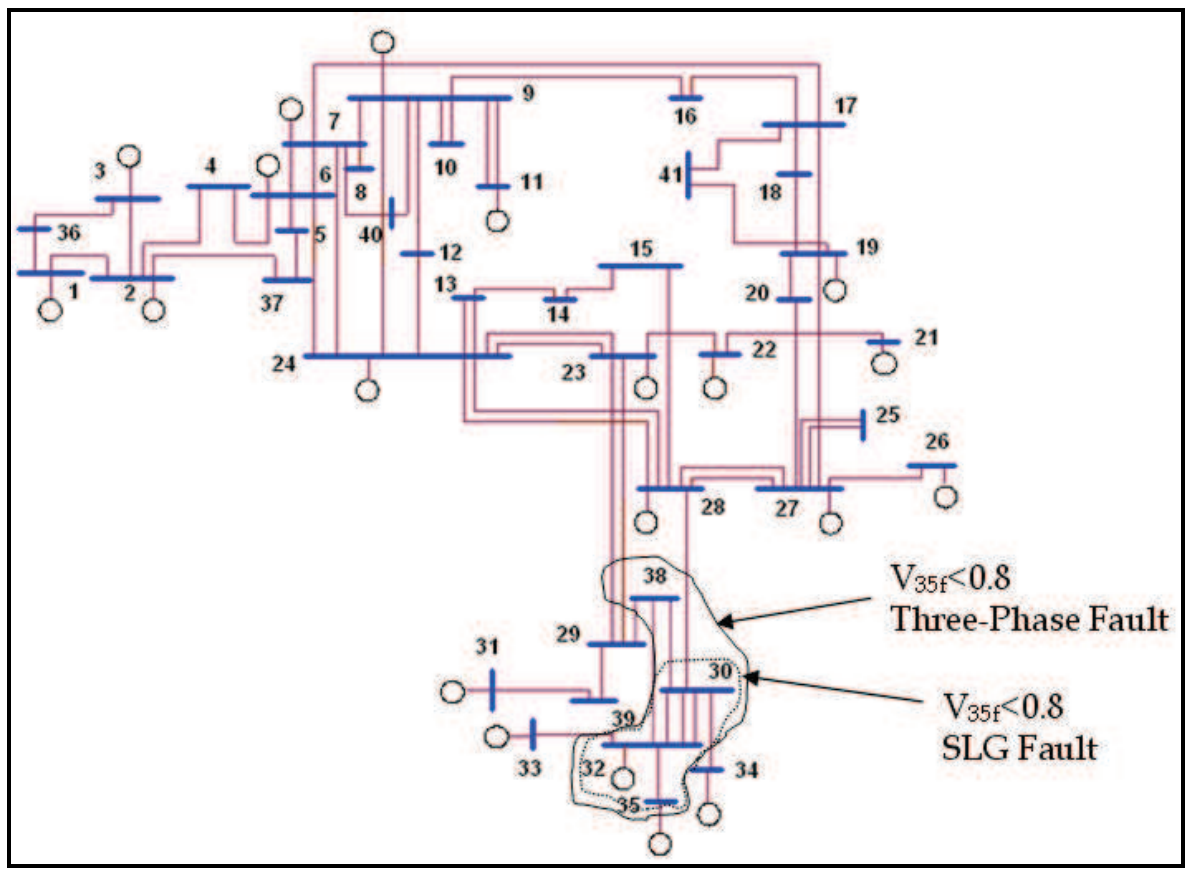

Fig. 6. Exposed area of bus 35 for SLG and three-phase faults

It is noted that the exposed area is also the area for which a monitor, installed at a particular bus $k$, is able to detect faults. For example, if a monitor is installed at bus 30 and the boundary for sag recording is adjusted to $0.8 \mathrm{pu}$, then the monitor is be able to see the faults in the $0.8 \mathrm{pu}$ exposed area of bus 30 . When referring to power quality monitors the exposed area is called Monitor Reach Area (MRA). In the next section, the way of optimal locating of the monitors is described to monitor all of the faults in the system.

\section{Optimal placement of voltage sag monitors}

In order to find optimal monitor locations for a monitoring program, the following two premises are considered:

- A minimum number of monitors should be used.

- No essential data concerning the performance of load buses in terms of sags should be missed.

The number of voltage sags recorded at a substation during a given monitoring time depends upon the critical threshold setting of the power quality monitor. It is considered the threshold level as the voltage $p$ in pu at which the monitor starts recording. If the threshold is set too low (e.g., $0.5 \mathrm{pu}$ ), then the monitor do not capture an important number of disturbances. On the other extreme, if the threshold $(p)$ is set high (e.g., 0.9 pu or higher) 
then the number of voltage sags recorded are excessive and even exceed the storage capability of the monitor. This increase or decrease in the number of events captured can be explained from the growth of the exposed area with increasing sensitivity of the monitor.

The MRA is defined as the area of the network that is observed from a given monitor position. The exposed area of a bus $k$ is exactly the monitor reach area of a monitor installed at that bus. The ability of the monitor to sense the remote faults is determined by the voltage threshold setting $p$. The MRA is greater for small voltage changes than for big ones. In other words, one monitor is theoretically able to capture all faults in the network for $p$ equal to 1 pu. Similarly, only solid faults occurring at the monitor position is seen for a threshold $p$ equal to $0 \mathrm{pu}$.

\subsection{Monitor Reach Area (MRA)}

The part of the network that is observed by a monitor installed at bus $k$ is thought as an area containing bus $k$ and its electrical neighbourhood. The size of this area depends mainly

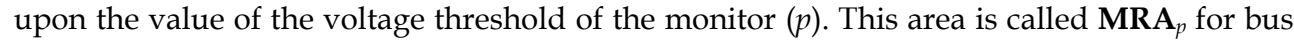
$k$. MRA $_{p}$ is the area of the transmission network, surrounding the monitor position $k$, for which the monitor is able to capture voltage drops that result in a retained voltage less than or equal to $p$ pu.

The $\mathbf{M R A _ { p }}$ of bus $k$ is shown as a set of buses. The $\mathbf{M R A _ { k , p }}$ is the set containing the indices of the buses within the $\mathbf{M R A _ { p }}$ of bus $k$. Using the voltage sag matrix $\mathbf{V}_{\mathrm{dfv}}, \mathbf{M R \mathbf { A } _ { k , p }}$ is determined as follows:

$$
\begin{aligned}
& \text { MRA }_{k, p}=\{k, i\} \quad i=1, \ldots, k, \ldots, n \\
& \text { subject to } \\
& v_{k i} \leq p \quad \forall k
\end{aligned}
$$

The MRA is easily determined from the rows of the voltage sag matrix. A given bus $i$ is part of the MRA of bus $k$ if the voltage at bus $k$ during a fault at bus $i$ is lower than or equal to $p$ pu. Equation (27) allows describing all the monitor reach areas and for any voltage threshold setting of the monitor.

An alternative method to describe the MRAs is by using a binary matrix. For a given voltage threshold setting $p$, the MRAs are described through an $n \times n$ binary matrix in which a 1 in entry $(i, j)$ indicates that bus $j$ belongs to the MRA of bus $i$. Equation (28) shows this matrix, where $v_{i j}$ is the $(i, j)$ entry of the voltage sag matrix.

$$
\mathbf{M R A}_{p}=\left[m r a_{i j}\right]=\left\{\begin{array}{ll}
1 & \text { if } v_{i j} \leq p \\
0 & \text { if } v_{i j}>p
\end{array} \quad \forall i, j\right.
$$

Once the MRAs for all possible locations are determined the optimization problem is formulated to locate the optimum number of the monitors.

\subsection{Optimization problem}

Consider a binary variable row vector $\mathbf{X}$ of length $n$ indicating if a monitor is needed at bus $i$. Each element of $\mathbf{X}, x_{i}$, is indicated as follows 


$$
x_{i}=\left\{\begin{array}{cc}
1 & \text { if monitor at bus } i \\
0 & \text { otherwise }
\end{array} \forall i\right.
$$

The vector $\mathbf{X}$ is called Monitor Position Vector (MPV). A given combination of ones and zeros indicates where to install the monitors as expressed in Equation (29).

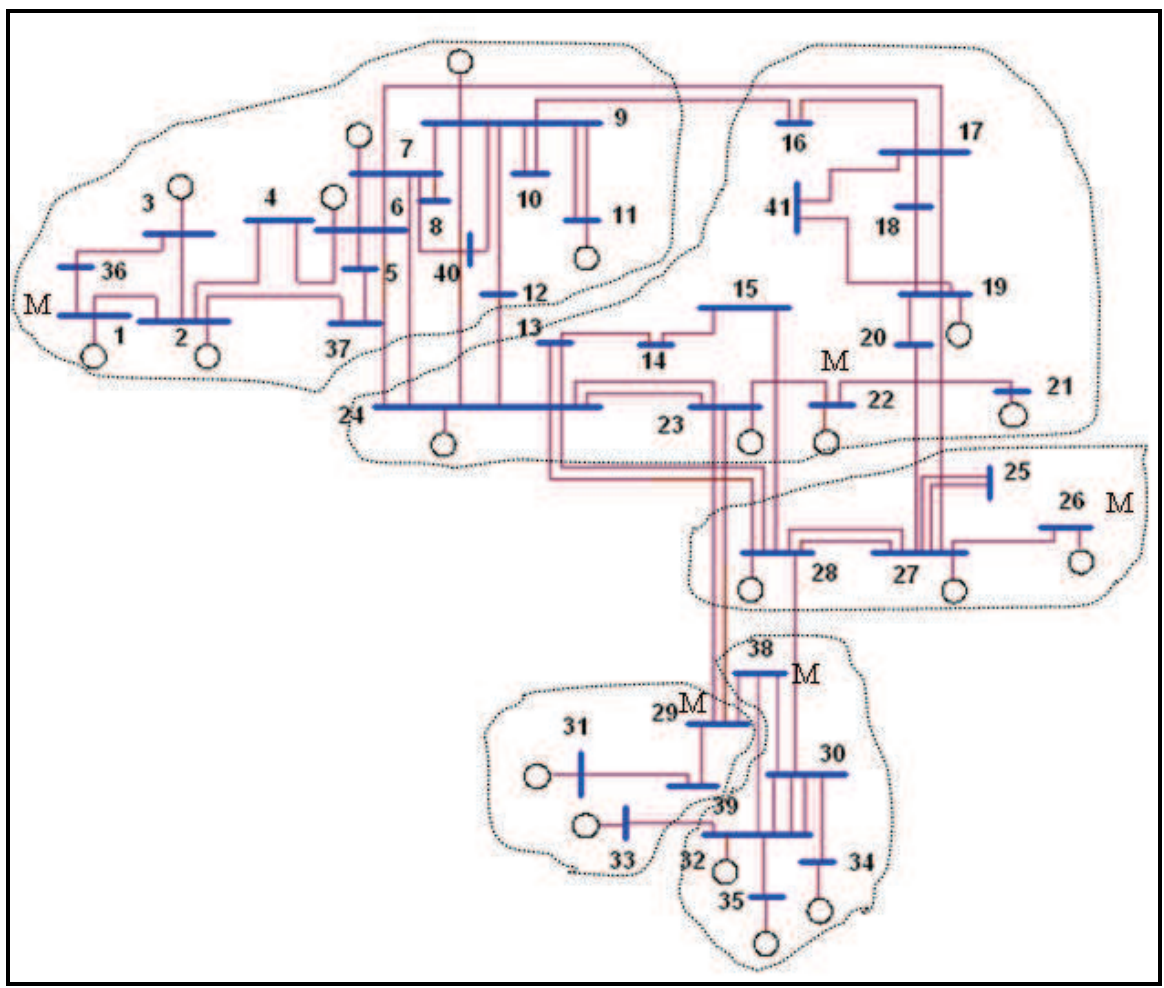

Fig. 7. Optimal Monitors Emplacements

It is noted that for a given value of the MPV the product of $\mathbf{X}$ by the $\mathbf{M R A}_{p}$ matrix indicates the number of MRAs that contain each one of the buses $i$. In order to satisfy the second premise, this product should be greater than or equal to 1 for each bus; meaning that each bus should be in at least one monitor reach area. Let $b$ be a row vector containing ones. The Objective Function $(O F)$ of the optimization problem is formulated as follows

$$
\begin{aligned}
& \min O F=\sum_{i=1}^{n} x_{i} \\
& \text { subject to } \\
& \sum_{i=1}^{n} x_{i} m r a_{i j} \geq b_{i}, \quad j=1,2, \ldots, n
\end{aligned}
$$

Where $x_{i}$ is the binary decision variable indicating the need for a monitor at bus $i ; n$ is the number of potential monitor positions. The right hand side, vector $b$, defines the level of 
redundancy of the monitoring program. A particular value of $b_{i}$ indicates that a fault at the fault position $i$ trigger at least $b_{i}$ monitors. The level of redundancy of the monitoring program is the minimum number of monitors that is guaranteed to trigger on the occurrence of a fault. The letter T over MRA $\mathbf{A}_{p}$ indicates transposition of the MRA matrix for voltage threshold $p$.

The voltage sag matrix can be used instead of the $\mathbf{M R A}_{p}$ matrix to formulate the optimization problem. This option allows modelling different voltage threshold setting for the monitors. The problem described by Equation (30) is an integer programming optimization problem. A number of algorithms, e.g. Branch and bound Algorithm (BBA) and Genetic Algorithm (GA), have been proposed for solving this type of problem.

As an example, the optimization method is applied to the sample network of Fig. 3. The BBA algorithm is used to solve the optimization problem. Let the voltage threshold $p$ of the monitors equals to $0.9 \mathrm{pu}$. If all of the faults are considered to be three phase faults, the results of the optimization show that the monitors should be installed at the buses 1, 22, 26, 29 , and 38. It is clear that the number of monitors needed to cover the whole system increases with the decrease of the monitor threshold. Fig. 7 shows the optimal monitors emplacements and their reach area.

In Fig. 7, letter $M$ shows the monitor places. The dashed lines also show the reach area of each of the monitors.

\section{Conclusion}

Power quality monitoring is necessary to characterize electromagnetic phenomena at a particular location on an electric power circuit. In this chapter, the monitoring of voltage sag which is one of the most important power quality phenomena has been discussed. The voltage sag magnitude has been monitored to find the origin of the voltage sag and detect all of the sags in the system.

Voltage sags are determined by fault types, fault impedances, and etc. With respect to the fault type, the shape of the rms voltage evolution shows different behavior. The calculations of all types of faults which may cause the sags have also been discussed in this chapter.

Ideally, a full monitoring program can be used to characterize the performance of entire system, i.e. every load bus should be monitored. Such a monitoring program is not economically justifiable and only a limited set of buses can be chosen for a monitoring program. This has led to the optimal monitoring program which has been proposed in this chapter.

\section{References}

Baggini, A. (2008). Handbook of Power Quality, Wiley-IEEE Press, ISBN 978-0-470-06561-7, John Wiley \& Sons Ltd, West Sussex, England

Bollen, M.H.J. (1999). Understating Power Quality Problems: Voltage Sags and Interruptions, Wiley-IEEE Press, ISBN 978-0-7803-4713-7, New-York, USA

Casarotto, C. \& Gomez, J.C. (2009). Calculation of Voltage Sags Originated in Transmission Systems Using Symmetrical Components, Proceedings of the 20th International Conference on Electricity Distribution (CIRED), Parague, June 8-11, 2009 
Gerivani, Y. ; Askarian Abyaneh, H. \& Mazlumi, K. (2007). An Efficient Determination of Voltage Sags from Optimal Monitoring, Proceedings of the 19th International Conference on Electricity Distribution (CIRED), Vienna, Austria, May 21-24, 2007

Grigsby, L.L. (2001). The Electric Power Engineering Handbook, CRC Press, ISBN 978-1-42003677-0, Florida, USA

Mazlumi, K.; Askarian Abyaneh, H. ; Gerivani, Y. \& Pordanjani, I.R. (2007). A New Optimal Meter Placement Method for Obtaining a Transmission System Indices, Proceedings of Power Tech 2007 conference, pp. 1165-1169, Lausanne, Switzerland, July 1-5, 2007

Milanovic, J.V.; Aung, M.T. \& Gupta, C.P. (2005). The Influence of Fault Distribution on Stochastic Prediction of Voltage Sags. IEEE Transactions on Power Delivery, Vol.20, No.1, (January 2005), pp. 278-285

Moschakis, M.N. \& Hatziargyriou, N.D. (2006). Analytical Calculation and Stochastic Assessment of Voltage Sags. IEEE Transactions on Power Delivery, Vol.21, No.3, (July 2006), pp. 1727-1734

Olguin, G. \& Bollen, M.H.J. (2002). Stochastic Prediction of Voltage Sags: an Overview, Proceedings of Probabilistic Methods Applied to Power Systems Conference, Naples, Italy, September 22-26, 2002

Olguin, G. (2005). Voltage Dip (Sag) Estimation in Power Systems based on Stochastic Assessment and Optimal Monitoring, Ph.D. Thesis, Chalmers University of Technology, Göteborg, Sweden

Olguin, G.; Bollen, M.H.J. (2002). The Method of Fault Position for Stochastic Prediction of Voltage Sags: A Case Study, Proceedings of Probabilistic Methods Applied to Power Systems Conference, Naples, Italy, september 22-26, 2002

Salim, F. \& Nor, K.M. (2008). Optimal voltage sag monitor locations, Proceedings of the Australasian Universities Power Engineering Conference (AUPEC '08), Sydney, Australia, December 14-17, 2008 


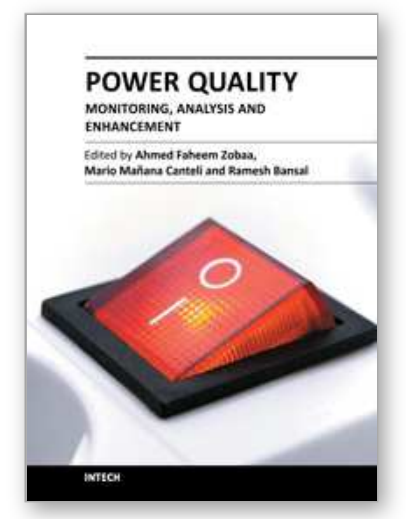

\author{
Power Quality â€“Â Monitoring, Analysis and Enhancement \\ Edited by Dr. Ahmed Zobaa
}

ISBN 978-953-307-330-9

Hard cover, 364 pages

Publisher InTech

Published online 22, September, 2011

Published in print edition September, 2011

This book on power quality written by experts from industries and academics from various counties will be of great benefit to professionals, engineers and researchers. This book covers various aspects of power quality monitoring, analysis and power quality enhancement in transmission and distribution systems. Some of the key features of books are as follows: Wavelet and PCA to Power Quality Disturbance Classification applying a RBF Network; Power Quality Monitoring in a System with Distributed and Renewable Energy Sources; Signal Processing Application of Power Quality Monitoring; Pre-processing Tools and Intelligent Techniques for Power Quality Analysis; Single-Point Methods for Location of Distortion, Unbalance, Voltage Fluctuation and Dips Sources in a Power System; S-transform Based Novel Indices for Power Quality Disturbances; Load Balancing in a Three-Phase Network by Reactive Power Compensation; Compensation of Reactive Power and Sag Voltage using Superconducting Magnetic Energy Storage; Optimal Location and Control of Flexible Three Phase Shunt FACTS to Enhance Power Quality in Unbalanced Electrical Network; Performance of Modification of a Three Phase Dynamic Voltage Restorer (DVR) for Voltage Quality Improvement in Distribution System; Voltage Sag Mitigation by Network Reconfiguration; Intelligent Techniques for Power Quality Enhancement in Distribution Systems.

\title{
How to reference
}

In order to correctly reference this scholarly work, feel free to copy and paste the following:

Kazem Mazlumi (2011). Power Quality Monitoring, Power Quality â€"Â Monitoring, Analysis and Enhancement, Dr. Ahmed Zobaa (Ed.), ISBN: 978-953-307-330-9, InTech, Available from:

http://www.intechopen.com/books/power-quality-monitoring-analysis-and-enhancement/power-qualitymonitoring

\section{INTECH}

open science | open minds

\author{
InTech Europe \\ University Campus STeP Ri \\ Slavka Krautzeka 83/A \\ 51000 Rijeka, Croatia \\ Phone: +385 (51) 770447 \\ Fax: +385 (51) 686166 \\ www.intechopen.com
}

\author{
InTech China \\ Unit 405, Office Block, Hotel Equatorial Shanghai \\ No.65, Yan An Road (West), Shanghai, 200040, China \\ 中国上海市延安西路65号上海国际贵都大饭店办公楼405单元 \\ Phone: +86-21-62489820 \\ Fax: +86-21-62489821
}


(C) 2011 The Author(s). Licensee IntechOpen. This chapter is distributed under the terms of the Creative Commons Attribution-NonCommercialShareAlike-3.0 License, which permits use, distribution and reproduction for non-commercial purposes, provided the original is properly cited and derivative works building on this content are distributed under the same license. 